\title{
A case of lung injury resembling diffuse pulmonary hemorrhage after the first administration of alemtuzumab in a patient with multiple sclerosis. Role of the HRCT
}

\author{
Gaia Cipolla ${ }^{1}$, Rosalba Relo${ }^{1}$, Elisa Pasciuta ${ }^{1}$, Domenica Catalano ${ }^{1}$, Federica Lo Bello ${ }^{1}$, \\ Irene Coppolino ${ }^{1}$, Paolo Ruggeri ${ }^{1}$, Alfio Proietto ${ }^{1}$, Maria Buccafusca ${ }^{2}$, \\ Paola Maria Cutroneo $^{3}$, Gianluca Trifirò ${ }^{3}$, Gaetano Caramori ${ }^{1}$ \\ ${ }^{1}$ Pulmonology, ${ }^{2}$ Neurology; ${ }^{3}$ Sicilian Regional Pharmacovigilance Centre, AOU Policlinico G. Martino, Messina, Italy
}

\begin{abstract}
Diffuse pulmonary hemorrhage (DPH) is an uncommon, acute condition characterized by a variable combination of hemoptysis, dyspnoea, anemia, hypoxemia, and an initial nonspecific imaging features such as diffuse and bilateral ground glass pulmonary opacities that can be induced by different causes. DPH is a rare manifestation of adverse drug reactions. We report here the case of a 25-year-old woman that has been admitted to our pulmonary clinic for the onset of chest pain, cough and haemoptysis, started one week after her first treatment with alemtuzumab for multiple
\end{abstract}

Correspondence: Gaetano Caramori, Pneumologia, Dipartimento di Scienze Biomediche, Odontoiatriche e delle Immagini Morfologiche e Funzionali (BIOMORF), Università degli Studi di Messina, 98122 Messina, Italy.

Tel. +39.090.2212000.

E-mail: gcaramori@unime.it

Key words: diffuse pulmonary haemorrhage; multiple sclerosis; alemtuzumab, adverse drug reaction.

Ethics approval: No ethical committee approval was required for this case report by the Department, because this article does not contain any studies with human participants or animals. Informed consent was obtained from the patient included in this study.

Consent for publication: The patient gave her written consent to use her personal data for the publication of this case report and any accompanying images.

Conflict of interest: The authors declare that they have no competing interests, and all authors confirm accuracy.

Received for publication: 30 April 2020.

Accepted for publication: 3 June 2020.

COpyright: the Author(s), 2020

Licensee PAGEPress, Italy

Monaldi Archives for Chest Disease 2020; 90:1352

doi: 10.4081/monaldi.2020.1352

This article is distributed under the terms of the Creative Commons Attribution Noncommercial License (by-nc 4.0) which permits any noncommercial use, distribution, and reproduction in any medium, provided the original author(s) and source are credited. sclerosis. Computed tomography (CT) scan of the chest at the admission showed diffuse and bilateral ground glass pulmonary opacities. Her symptoms resolved completely without any treatment, after the interruption of alemtuzumab, and a CT scan of the chest performed one month later showed total disappearance of the pulmonary opacities.

\section{Introduction}

Diffuse pulmonary hemorrhage (DPH) is a clinical syndrome resulting from the accumulation of erythrocytes into the alveolar space as a result of injury to the microcirculation of the lung $[1,2]$; it is characterized by a variable combination of hemoptysis, dyspnoea, anemia, hypoxemia and an initial nonspecific imaging features such as diffuse and bilateral ground glass pulmonary opacities [3]. It can be induced by different causes, including adverse reaction to several drugs (Table 1).

Alemtuzumab is a humanized, rat monoclonal immunoglobulin (Ig) G1 antibody with a molecular weight of $\sim 150 \mathrm{kDa}$, anti-cluster of differentiation 52 (CD52) [4], a membrane glycoprotein expressed on T and B lymphocytes, natural killer cells, monocytes, macrophages, dendritic cells, and eosinophilic granulocytes. CD52 is involved in T lymphocyte co-stimulation, induction of regulatory $\mathrm{T}$ lymphocytes, and T lymphocyte migration and adhesion [5]. Alemtuzumab causes a rapid depletion of circulating $\mathrm{T}$ and $\mathrm{B}$ lymphocytes, as well as various cells of the innate immune system, for this reason, it is used for the treatment of patients with active relapsing-remitting multiple sclerosis [6].

\section{Case Report}

We present here the case of a 25-year-old Caucasian woman (height $161 \mathrm{~cm}$, weight $45 \mathrm{Kg}$, body mass index $17.4 \mathrm{Kg} / \mathrm{m}^{2}$ ), who has been admitted to our pulmonary clinic from the emergency ward of our hospital, in September 2018, for the acute onset of chest pain, dyspnoea at rest, cough and non massive haemoptysis $(<10$ $\mathrm{mL}$ ) [7], started after the fourth dose of alemtuzumab (intravenous injection of $12 \mathrm{mg}$ once daily) administered for a relapse of multiple sclerosis. Alemtuzumab was administered for the first time on the $13^{\text {rd }}$ September of 2018 , followed by other three doses, respectively on $14^{\text {th }}, 16^{\text {th }}$ and $17^{\text {th }}$ September of 2018 . After her $3^{\text {rd }}$ dose of the treatment, mild erythema appeared in the upper limbs and the trunk 
required treatment with oral histamine 1 receptor antagonist (intravenous injection of chlorphenamine $10 \mathrm{mg}$ ). She stopped the treatment with alemtuzumab at the occurrence of the pulmonary symptoms.

She was unemployed, a non-smoker and had a medical history of epilepsy (diagnosed in May 2015) secondary to the presence of a cavernous angioma of the left frontal lobe that has been treated in 2015 with local embolization, and then followed by a regular therapy with levetiracetam (500 mg once daily). In January 2017 she has been diagnosed multiple sclerosis, after performing, in October 2016, a somatosensory evoked potential test (showed an altered nerve impulse propagation along central somatosensory pathways), and in November 2016 a spine magnetic resonance imaging scan (showing demyelinating lesions in the white dorsal and cervical spinal cord).

At the admission in our clinic, her vital signs were: systemic blood pressure 120/85 $\mathrm{mmHg}$, pulse frequency 80/min (rhythmic), body axillary temperature $36.8^{\circ} \mathrm{C}$, oxygen pulse oximetry value of $98 \%$ (when breathing room air) with a respiratory rate of 22 breaths per min. Physical examination was normal. Computed tomography
(CT) scan of the chest showed diffuse and bilateral ground glass pulmonary opacities, without pleural effusions (Figure 1A). During her hospitalization, the routine laboratory examinations showed a mild reduction of serum haemoglobin value of $13.5 \mathrm{~g} / \mathrm{dL}$, compared to the last available value of $14.6 \mathrm{~g} / \mathrm{dL}$ referred to the $11^{\text {th }} \mathrm{June}$ of 2018 obtained from patient's past medical records. A mild increase of the serum C-reactive protein value $(2.27 \mathrm{mg} / \mathrm{dL}$, normal values $<0.5 \mathrm{mg} / \mathrm{dL}$ ) was also reported. The diffusing capacity for carbon monoxide (Figure 2A), performed on the $4^{\text {th }}$ day after hospitalization, showed a normal value when corrected for actual hemoglobin level ( $85 \%$ of the predicted value). The intradermal reaction to purified protein derivative skin test was negative. The search for serum $\operatorname{IgG}$ and $\operatorname{IgM}$ antibodies anti-Mycoplasma pneumoniae, Chlamydophila pneumoniae, and Epstein-Barr virus were negative; as well as the urinary Legionella pneumophila and Pneumococcal antigens; and the serum and urine research of DNA of Cytomegalovirus was negative. An echocardiogram was normal with a systolic ejection fraction of $60 \%$ and a normal value of systemic pulmonary arterial pressure. An ear, nose and throat examination with endoscopy of the upper airways did not show any

Table 1. List of drugs inducing DPH. Antiplatelet agents

Abciximab, cilostazol, clopidogrel, epoprostenol, epifibatide, ticagrelor, ticlopidine, tirofiban Anticoagulants and thrombolytic drugs

Apixaban, alteplase, brodifacoum, dabigatran, edoxaban, enoxaparin, rivaroxaban, streptokinase

Nonsteroidal anti-inflammatory and other anti-inflammatory drugs

Acetylsalicylic acid, azathioprine, ketorolac, oxyphenbutazone, paracetamol, penicillamine, systemic corticosteroids

Anti-infective drugs

Azithromycin, itraconazole, levamisole, metronidazole, moxalactam, nitrofurantoin, quinine

Antiarrhythmic and antihypertensive drugs

Amiodarone, hydralazine, ivabradine, quinidine

Antithyroid and other endocrinological drugs

Aminoglutethimide, clomiphene, ergometrine, methimazole, propylthiouracil Oncological drugs

All-transretinoic acid, arsenic trioxide, bartezomib, carfilzomib, crizotinib, cyclophosphamide, cytarabine, docetaxel, erlotinib, etoposide, everolimus, fludarabine, gefitinib, gemcitabine, imatinib, irinotecan, lenalidomide, methotrexate, mytomicin c, nilotinib, pemetrexed, sunutinib

Immunosuppressive drugs

Mycophenolate mofetil, sirolimus

Monoclonal antibodies

Alemtuzumab, bevacizumab, etanercept, gemtuzumab, infliximab, nivolumab, rituximab

Oppioids

Fentanyl, morphine

Antiepileptic drugs

Phenytoin, valproic acid

Herbal medicines

Ginkgo, ginseng, makyo-kanseki-to, sho seiryu-to, seisin-renshi-in

Other drugs

Benzbromarone, chlorphenamine, hyaluronic acid, lipiodol, montelukast, sevoflurane, sildenafil

Data from Pneumotox.com and Pubmed https:/www.ncbi.nlm.nih.gov/pubmed/ 
lesion, and an infectivology consultation did not recommend any antibiotic therapy. The patient refused to perform bronchoscopy with bronchoalveolar lavage.

She was left untreated and no other episodes of haemoptysis occurred during her hospitalization. The patient was discharged at home after nine days. After one month she returned to our outpatient clinic for a post-discharge visit and performed a test to evaluate the diffusion capacity for carbon monoxide (Figure 2B), that showed a normal value ( $119 \%$ of the predicted value). A new CT scan of the chest, performed on the $20^{\text {th }}$ October 2018 , showed the complete resolution of the pulmonary opacities (Figure 1B). All these data made us lean towards a diagnosis of diffuse pulmonary hemorrhage.

\section{Discussion}

DPH is a complex syndrome that may represent a diagnostic and therapeutic challenge, with potentially significant morbidity and mortality. For this reason, the identification of the underlying cause of DPH with the initiation of appropriate treatment, whenever possible, is essential for improving prognosis $[8,9]$. Imaging features of DPH depend on its temporal evolution. At presentation, chest imaging is normal in $20 \%-50 \%$ of cases. When present initial radiographic findings consist of patchy ground glass opacities with a central and basilar predominance and sparing the costophrenic angles. The subacute phase (within $48 \mathrm{~h}$ ) is generally characterized by developing of interlobular and intralobular interstitial thicknening associated with the persistence of ground glass opacities by configuring the crazy-paving pattern on chest computed tomography. When DPH is chronic and recurrent, pulmonary fibrosis develops with regions of architectural distortion and areas of lobular sparing [1]. DPH can be clinically categorized into vasculitis, pulmonary-renal syndromes, autoimmune diseases, druginduced, and idiopathic. Drug-induced DPH imaging is typically characterized by diffuse bilateral ground-glass opacities in the acute setting with identification at the medical history of a clear causeeffect relationship [1]

Laboratory findings during DPH often demonstrate anemia, leukocytosis, levels elevated erythrocyte sedimentation rate and serum $\mathrm{C}$ reactive protein; however, the absence of these findings does not exclude DPH. Moreover, initial laboratory data typically show anemia especially in patients with subacute or chronic bleeding instead of acute bleeding as in our case [1,3]. Increased uptake of carbon monoxide may classically help in the diagnosis of DPH when recent (48-72 hours), especially when combined with a restrictive pattern. The diffusing capacity for carbon monoxide in our patient was within normal value when corrected for actual hemoglobin level. The diffusing capacity for carbon monoxide is not a reliable diagnostic tool, often it cannot be performed in the acute settings, and it requires repetitive measurement for confirmation because of a wide normal range [10]. In January 2020 a National Drug Authority (AIFA) note introduced for alemtuzumab new therapeutic limitations and
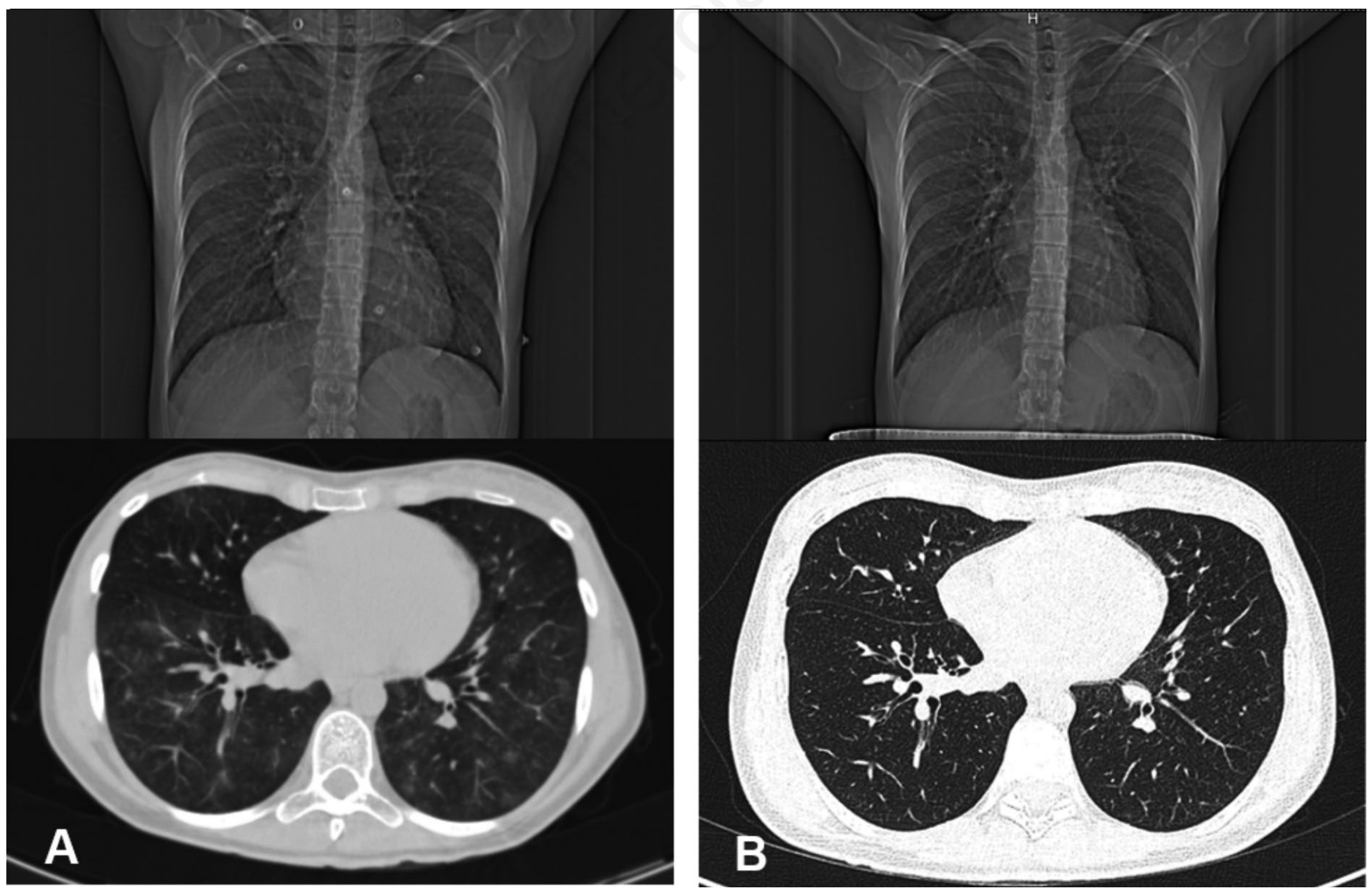

Figure 1. A) Chest computed tomography scan at the hospital admission showing diffuse and bilateral ground-glass pulmonary opacities. B) Chest computed tomography scan performed one month after the admission showed complete resolution of the opacities. 




Figure 2. A) The diffusing capacity for carbon monoxide test performed after four days of hospitalization showed a normal value. B) The diffusing capacity for carbon monoxide test performed one month after the discharge showed a normal value.

contraindications, for the retrieving of severe adverse reactions, sometimes fatal [11].

In our patient DPH as manifestation of drug effect may be higher on the list of diagnostic considerations at the light of the imaging findings and their temporal evolution with their resolution one month after drug withdrawal. We performed a careful differential diagnostic approach, with alemtuzumab resulting the most likely cause of her DPH. The only other drug administered was chlorphenamine, and no adverse pulmonary reactions are reported in the product information of AIFA or MicroMedex database, while only 4 cases of pulmonary haemorrhage are reported in Vigibase [12].

The mechanisms of drug induced DPH are heterogeneous including autoimmune or hypersensitivity reactions, or direct damage of the alveolar epithelium or of its basement membrane [13]. The mechanism by which alemtuzumab causes DPH is unknown. We performed a search of some database of suspected adverse drug reactions in VigiBase, the World Health Organization's database [12], and we found record of 16,517 cases of adverse alemtuzumab reactions, of which 27 of pulmonary alveolar haemorrhage, 20 of pulmonary haemorrhage and 5 of diffuse alveolar damage; while the European Medicines Agency database, Eudravigilance [14], reported 7072 adverse alemtuzumab reactions cases, of which 4 of diffuse alveolar haemorrhage, 17 of pulmonary alveolar haemorrhage and 12 of pulmonary haemorrhage. According to Naranjo causality assessment scale [15] the causal relationship between use of alemtuzumab and DPH was scored as "probable".

Two cases of alemtuzumab associated diffuse alveolar hemorrhage (DAH) in patients with multiple sclerosis have been previously reported in literature [16,17]. As in our report, they were two young women that developed acute dyspnoea, chest pain and haemoptysis during first cycle of alemtuzumab $12 \mathrm{mg}$; body temperature, blood pressure and heart rate were normal; laboratory routine examination showed normal values. In Myro case report [16], the patient showed an increased value of serum $\mathrm{C}$ reactive protein $(2.6 \mathrm{mg} / \mathrm{dL})$ as our patient, and haemoglobin fell from 12 $\mathrm{g} / \mathrm{dL}$ to $10.5 \mathrm{~g} / \mathrm{dL}$ after the administration of alemtuzumab. In both cases, CT scan of the chest showed diffuse and bilateral ground glass pulmonary opacities with centrilobular distribution. In Myro case [16] no treatment was given, and the symptoms resolved spontaneously in two days, while in Bianco report [17], the patient was treated with methylprednisolone IV $1000 \mathrm{mg} /$ day. In both patients CT performed after hospitalization showed total resolution of pulmonary opacities.

\section{Conclusions}

Despite the mechanism by which alemtuzumab causes DPH is unknown, we suggest to all the clinicians prescribing alemtuzumab to consider DPH as a potential complication of this treatment. Imaging maintains a central role in arriving at the diagnosis of DPH considering distinguishing features and their temporal evolution.

\section{References}

1. Lichtenberger JP III, Digumarthy SR, et al. Diffuse pulmonary hemmorrhage: clues to the diagnosis. Curr Probl Diagn Radiol 2014;43:128-39.

2. Lara AR, Schwarz MI. Diffuse alveolar hemorrhage. Chest 2010;137:1164-71.

3. Nasser M, Cottin V. Alveolar haemorrhage in vasculitis (primary and secondary). Semin Respir Crit Care Med 2018;39: 482-93.

4. Baan CC, Gelder T, Hesselink DA. Review of the clinical pharmacokinetics and pharmacodynamics of alemtuzumab and its use in kidney transplantation. Clin Pharmacokinet 2018; 57 : 191-207.

5. Ambrose LR, Morel AS, Warrens AN. Neutrophils express CD52 and exhibit complement-mediated lysis in the presence of alemtuzumab. Blood 2009;114:3052-55.

6. Havrdova E, Harakova D, Kavarova I et al. Alemtuzumab in the treatment of multiple sclerosis: key clinical trial results and considerations for use. Ther Adv Neurol Disord 2015;8:31-45.

7. Bidwell JL, Pachner RW. Hemoptysis: diagnosis and management. Am Fam Physician 2005;72:1253-60.

8. Rossi SE, Erasmus JJ, McAdams HP, et al. Pulmonary drug toxicity: radiologic and pathologic manifestations. Radiographics 2000;20:1245-59. 
9 . De Prost N, Parrot A, Cuquemelle E, et al. Diffuse alveolar hemorrhage in immunocompetent patient: etiologic and prognosis revisited. Respir Med 2012; 106:1021-32

10. Ioachimescu OC, Stoller JK. Diffuse alveolar hemorrhage: diagnosing it and finding the cause. Cleve Clin J Med 2008;75: 258-65.

11. Agenzia Italiana del Farmaco (AIFA). [Nota Informativa Importante su Lemtrada (alemtuzumab)].[in Italian]. Accessed on: 6 March 2020. Available from: https:// www.aifa.gov.it/-/nota-informativa-importante-su-lemtradaalemtuzuma-2

12. VigiBase. The World Health Organization international database of suspected adverse drug reactions. Accessed on: 6 March 2020. Available from: http://www.vigiaccess.org/

13. Schwarz MI, Fontenot AP. Drug-induced diffuse alveolar haemorrhage syndromes and vasculitis. Clin Chest Med 2004;25:133-40.

14. EudraVigilance. European database of suspected adverse drug reaction reports. ccessed on: 6 March 2020. Available from: http://www.adrreports.eu/index.html

15. Naranjo CA, Busto U, Sellers EM, et al. A method for estimating the probability of adverse drug reactions. Clin Pharmacol Ther 1981;30:239-45.

16. Myro AZ, Bjerke G, Zarnovicky S, Holmoy T. Diffuse alveolar hemorrhage during alemtuzumab infusion in a patient with multiple sclerosis: a case report. BMC Pharmacol Toxicol 2018;19:75.

17. Bianco A, Mari P, Larici AR. Alemtuzumab-induced lung injury in multiple sclerosis: learning from adversity in three patients. Mult Scler Relat Dis 2020;37:101450. 